\title{
Composition of massive giant planets
}

\author{
Ravit Helled ${ }^{1}$, Peter Bodenheimer ${ }^{2}$ and Jack J. Lissauer ${ }^{3}$ \\ ${ }^{1}$ Department of Earth and Space Sciences, \\ University of California, Los Angeles, CA 90095-1567, USA, \\ ${ }^{2}$ University of California, Santa Cruz, CA 95064, USA \\ ${ }^{3}$ NASA-Ames Research Center, Moffett Field, CA 94035, USA \\ email: rhelled@ucla.edu or r.helled@gmail.com
}

\begin{abstract}
The two current models for giant planet formation are core accretion and disk instability. We discuss the core masses and overall planetary enrichment in heavy elements predicted by the two formation models, and show that both models could lead to a large range of final compositions. For example, both can form giant planets with nearly stellar compositions. However, low-mass giant planets, enriched in heavy elements compared to their host stars, are more easily explained by the core accretion model. The final structure of the planets, i.e., the distribution of heavy elements, is not firmly constrained in either formation model.
\end{abstract}

Keywords. planets and satellites: formation, planetary systems: formation

\section{Introduction}

The topic of giant planet formation has been studied for decades. The discoveries of giant planets outside our solar system have given us the opportunity to test existing formation and interior models of gas giant planets. Transiting extrasolar giant planets provide important information about their bulk compositions, and a large range of compositions for these objects has been deduced (Guillot 2008). Observations of extrasolar gas planets have raised many challenges for giant planet formation theories, including the determination of planetary formation location, formation timescale, and planetary composition and structure. The interplay between theories and observations is expected to provide a clearer and more complete picture of giant planet formation.

The standard model for giant planet formation, 'core accretion', is based on the hypothesis that the formation of a giant planet begins with planetesimal coagulation and heavy-element core formation, followed by accretion of a gaseous envelope (Pollack et al. 1996; Lissauer et al. 2009). A second model for giant planet formation is 'gravitational (disk) instability' in which gas giant planets form as a result of gravitational fragmentation in the disk surrounding the young star (Boss 1997; Durisen et al. 2007).

There are substantial differences between the two formation models, including formation timescale, the most favorable formation location, and the ideal disk properties for planetary formation. The different nature of the two formation mechanisms naturally leads to some expected differences in planetary composition. However, the final composition of a planet formed by each of these models depends upon the local disk properties in the region of planetary birth. As a result, no clear-cut criterion regarding planetary composition can be used to discriminate between these two formation mechanisms. Below we discuss the ranges of planetary compositions predicted by the two models, and their possible differences. 


\section{Core Accretion}

In the core accretion model, first, dust grains accrete to form solid planetesimals that merge to form a solid core surrounded by a thin gaseous atmosphere. Solids, which typically consist of rocks, ices and organics, are accreted on a time scale of 1 to a few Myr, while the gas accretion rate initially falls well below the solid accretion rate. The solid accretion rate decreases significantly once the planetesimals in the planet's feeding zone are depleted, while the gas accretion rate increases steadily. Eventually, the gas accretion rate exceeds the accretion rate of solid planetesimals, and gas continues to be accreted at a nearly constant rate. Once the core mass and the mass of the gaseous envelope become about equal, a runaway gas accretion builds up the mass of the envelope rapidly while leaving the core at a nearly constant value. Gas accretion then stops either by dissipation of nebular gas or by gap opening (Lissauer et al. 2009). After that point the planet is practically isolated from the disk, and it contracts quasi-statically and cools on a time scale of $10^{9}$ years (see Lissauer \& Stevenson 2007 and references therein).

\subsection{Core Mass}

The core masses of giant planets formed by core accretion can vary from a few Earth masses $\left(\mathrm{M}_{\oplus}\right)$ up to even tens of $\mathrm{M}_{\oplus}$. The growth of the core continues as long as planetesimals are present in the planet's feeding zone. The planet's feeding zone represents the region around the planet from which it can capture planetesimals, and its extent is normally taken to be about 4 Hill sphere radii inward and outward from the planet's orbit. The Hill sphere radius of the protoplanet is $R_{H}=a\left(\frac{M_{p}}{3 M_{\star}}\right)^{1 / 3}$ where $a$ is the planet's semimajor axis, $M_{p}$ is the planet's mass, and $M_{*}$ is the mass of the star. Once the feeding zone is depleted, the planetary object is nearly isolated and planetesimal accretion drops significantly. The mass at which isolation occurs is given by

$$
M_{i s o} \approx \frac{64}{\sqrt{3}} \pi^{3 / 2} M_{\star}^{-1 / 2} \sigma^{3 / 2} a^{3}
$$

where $\sigma$ is the solid surface density (Lissauer 1993). Around a $1 \mathrm{M}_{\odot}$ star at $5.2 \mathrm{AU}$ with $\sigma=10 \mathrm{~g} \mathrm{~cm}^{-2}$, this mass is about $11.5 \mathrm{M}_{\oplus}$.

The final core mass $M_{c} \approx \sqrt{2} M_{\text {iso }}$ (Pollack et al. 1996) because the feeding zone for planetesimals expands during the slow gas accretion phase. Equation (2.1) relates the core mass and disk properties provided that neither the planet nor the planetesimals migrate substantially. First, the isolation mass increases with radial distance, so planets in wider orbits are predicted to have more massive cores. Second, the isolation mass is proportional to $\sigma^{3 / 2}$ (Lissauer 1987). The more solids available to the growing planet, the more massive the core can become. It is therefore clear that the core mass would increase with increasing $\sigma$, and that for a given disk mass (or metallicity), steeper density profiles would result in massive cores at smaller radial distances (see Helled \& Schubert 2009 for details). Finally, $M_{i s o}$ is inversely proportional to the square root of stellar mass. Note that Movshovitz et al. (2010) find that core accretion can produce planets with cores of only a few earth masses.

\subsection{Planetary Composition}

The core accretion model does not predict a specific composition for the forming planet. The total heavy-element enrichment of the planet depends on (1) the planetary core mass, which, as just discussed, depends on disk properties, (2) on the amount of dust entrained within the accreted gaseous envelope, and (3) planetesimals (or even other planets) accreted subsequent to rapid gas accretion. 
The total mass fraction of heavy elements in the planet can by given by $Z=\left(M_{c}+\right.$ $\left.M_{Z_{e n v}}\right) / M_{p}$, where $M_{Z_{e n v}}$ is the mass of heavy elements in the gaseous atmosphere. This simple relation demonstrates the dependence of the bulk composition on both the core mass and the abundance of the accreted gas. The relation implies that the final composition of a massive planet is strongly dependent on the accreted gas composition.

For relatively massive planets, the core contains only a very small fraction of the total mass. The predicted planetary $Z$ depends on the accreted gas composition which could be that of the star, or dust-free or dust-enriched relative to the star. This point is illustrated in Fig. 1a, for different values of $M_{c}$, assuming that the composition of the gaseous envelope is solar. However, planets which are depleted in heavy elements compared to their host star could form as well. The gaseous envelope that is accreted could be depleted with solids

leading to a sub-stellar $Z$. Giant planets that are enriched with heavy elements compared to the star could form when cores are massive, when the accreted gas is enriched with solids, and when planetesimals are accreted at later stage of the planetary growth. It is therefore clear that the core accretion model could lead to the formation of planets with a wide range of final masses and compositions.
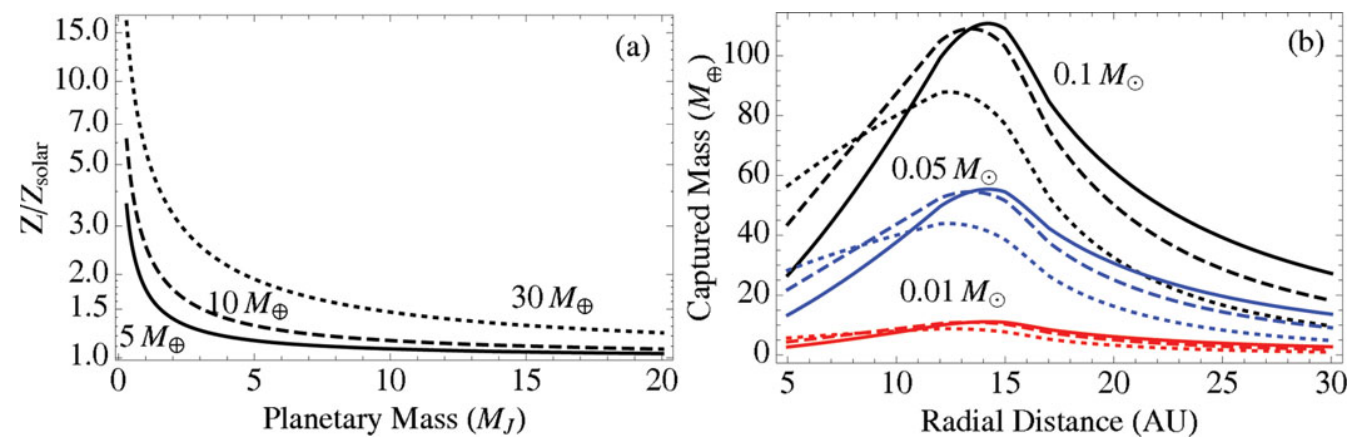

Figure 1. (a): metallicity of the planet divided by solar metallicity as a function of planetary mass for three different core masses: 5,10 , and $30 \mathrm{M}_{\oplus}$ (dotted, dashed, and solid curves, respectively) assuming a solar composition for the gas. (b): Captured solid mass by a planet formed by gravitational instability, as a function of the distance of the planet from the star. The black, blue and red curves are for disk masses of $0.1,0.05$, and $0.01 \mathrm{M}_{\odot}$, respectively. The solid, dashed, and dotted curves refer to density distributions proportional to $a^{-1 / 2}, a^{-1}$ and $a^{-3 / 2}$, respectively (Helled \& Schubert 2009).

\section{Gravitational Instability}

In the disk-instability model, planets are formed by fragmentation of the gas disk due to gravitational instabilities. Once a local instability occurs, a gravitationally bound subcondensation region can be created. The fragment contracts, and eventually evolves to become a giant planet. Numerical investigations suggest that planets in wide orbits, such as those recently observed by direct imaging (Marois et al. 2008), could form by disk instability.

The evolution of planets formed by gravitational instability is often followed using standard stellar (planetary) evolution equations, under the approximation of spherical symmetry. The model typically takes the objects to be gravitationally bound, isolated, homogenous and static, with stellar composition (Bodenheimer et al. 1980; Helled et al. 2006). 
The first evolutionary stage is the 'pre-collapse' stage in which the extended $(\approx 0.5$ AU) and cold (internal temperatures $<2000 \mathrm{~K}$ ) protoplanet contracts quasi-statically. The duration of this stage is not well constrained but as a first approximation it can be taken to be inversely proportional to the square of the initial mass of the body (Helled \& Bodenheimer 2011), so more massive protoplanets evolve faster. The pre-collapse stage ends when molecular hydrogen starts to dissociate at the center of the body and a hydrodynamic collapse ensues (Bodenheimer et al. 1980).

\subsection{Planetary Composition}

The initial composition of giant planets formed by gravitational instability is similar to the young disk's composition and is therefore stellar. However, the planet may subsequently be enriched in heavy elements by planetesimal capture (Helled et al. 2006; Helled \& Schubert 2008).

The amount of solid mass available for capture depends on the solid surface density $\sigma$ at the planetary location. As in the core accretion model, $\sigma$ changes with the disk mass and its radial density profile, and with stellar metallicity. The total available mass of solids in the planet's feeding zone can be given by

$$
M_{a v}=16 \pi a^{2}\left(\frac{M_{p}}{3 M_{\star}}\right)^{1 / 3} \sigma .
$$

Eq. (3.1) shows that the available mass for capture on one hand increases with radial distance due to its dependence on $a^{2}$, but also decreases with radial distance due to its dependence on $\sigma$, which decreases as a function of $a$. It is therefore clear that the mass available for capture strongly depends on the planetary semimajor axis $a$, and the disk properties (Helled \& Schubert 2008). Also, the available mass depends weakly on $M_{p}$. The available mass for accretion provides an upper limit to the enrichment for given conditions assuming that the planetesimals and/or the protoplanet do not migrate substantially. However, the actual enrichment depends on the ability of the planet to capture these solids during its pre-collapse contraction.

The planetesimal accretion rate is given by Safronov (1969)

$$
\frac{d m}{d t}=\pi R_{c a p}^{2} \sigma \Omega F_{g}
$$

where $R_{\text {cap }}$ is the protoplanet's capture radius, $\Omega$ is the protoplanet's orbital frequency, and $F_{g}$ is the gravitational enhancement factor. Eq. (3.2) suggests that the accretion rate is significantly smaller at large radial distances due to its dependence on both $\sigma$ and $\Omega$. As a result, giant planets at wide orbits will have insufficient time for accretion, which leads to negligible enrichment of solids (Helled \& Bodenheimer 2010). The protoplanet's capture radius depends on the planetary size and density, and is therefore larger with increasing planetary mass. Finally, the accreted mass depends on the available time for accretion. As discussed in Helled \& Schubert (2008), enrichment is efficient as long as the protoplanet is extended and fills most of the area of its feeding zone, so planetesimals can be slowed down by gas drag, and be absorbed by the protoplanet. Therefore the longer the pre-collapse stage is, the longer the time available for accretion; thus low mass protoplanets have more time to accrete solids, although the mass available for accretion is limited because of its dependence on the total planetary mass.

The planetary enrichment can therefore change significantly from one system to another. Helled et al. (2006) have shown that a Jupiter-mass $\left(\mathrm{M}_{J}\right)$ clump formed at 5.2 AU could have accreted more than $40 \mathrm{M}_{\oplus}$ of heavy elements. Helled \& Schubert (2009) have shown that a $1 \mathrm{M}_{J}$ protoplanet formed between 5 and $30 \mathrm{AU}$ could accrete $1-110 \mathrm{M}_{\oplus}$ of 
heavy elements, depending on disk properties, and concluded that in the disk instability model the final composition of a giant planet is strongly determined by its formation environment. Fig. 1b shows the heavy element enrichment found by Helled \& Schubert (2009). The figure presents the captured mass in the first $10^{5}$ years of the planetary evolution for all the cases considered.

Helled \& Bodenheimer (2010) have found that protoplanets with masses between 3 and $7 \mathrm{M}_{J}$ in wide orbits (24 to $68 \mathrm{AU}$ ) can accrete between tens and practically zero $\mathrm{M}_{\oplus}$, with the negligible mass corresponding to the larger radial distances. In the disk instability model, it is predicted that the planetary enrichment with heavy elements strongly decreases with radial distance, and that giant planets in wide orbits would have nearly stellar composition.

\subsection{Core Mass}

In the disk instability model the newly formed protoplanets have no cores. However, cores could form by settling of solids towards the planetary center (DeCampli \& Cameron 1979). Helled et al. (2008) presented a detailed analysis of coagulation and sedimentation of silicate grains in an evolving $1 \mathrm{M}_{J}$ protoplanet including the presence of convection. It was found that during the initial contraction of the protoplanet (pre-collapse stage), which lasts several times $10^{5}$ years, silicate grains can sediment to form a core both for convective and non-convective envelopes, although the sedimentation time is substantially longer if the envelope is convective. Grains made of ices and organics were found to dissolve in the planetary envelope, not contributing to core formation.

Helled \& Schubert (2008) have investigated the topic of core formation for protoplanets with masses between 1 Saturn mass and $10 \mathrm{M}_{J}$. It was found that grain settling occurs in low-mass protoplanets which are cold enough and have a contraction time-scale long enough for the grains to grow and sediment to the center. The grain sedimentation process was found to be favorable for low-mass bodies, due to lower internal temperatures, lower convective velocities and longer contraction time-scales. In convective regions, the grains are carried by the convective eddies until they grow to sizes of $10 \mathrm{~cm}$ or larger (the exact value depends on the convection velocity which changes with depth, time and planetary mass). Then they are massive enough to decouple from the gas, sediment to the center and form a core. Protoplanets with masses of $\geqslant 5 \mathrm{M}_{J}$ were found to be too hot to allow core formation. In this mass range the grains evaporate in the planetary envelope, enriching it with refractory material (see Helled \& Schubert, 2008 for details).

As a result, in the disk instability model, $M_{c}$ is not simply proportional to the mass of the protoplanet. Low-mass clumps have the lowest convective velocities and longest evolution-properties that support core formation. The final core mass depends on the available solid mass within the planet's envelope. If a substantial amount of solids can be accreted before internal temperature are high enough to evaporate the grains, cores of several $\mathrm{M}_{\oplus}$ could be formed (Helled et al. 2006; Helled \& Schubert 2008).

\section{Internal Structure}

Neither model for giant planet formation can provide tight constraints on the planetary internal structure. In core accretion, one prediction would be that the core consists of rocks, organics, and ices, while in the disk instability model the cores would be composed of refractory material. Thus, if large pieces of ice and organic material enter the planetary envelope, they could settle to the core as well (Helled et al. 2006; Helled \& Schubert 2008). In addition, core accretion simulations show that once the core mass reaches $\sim 3$ $\mathrm{M}_{\oplus}$, volatiles in small solid bodies dissolve in the envelope and do not reach the core, 
while for simplification, the model typically assumes that the accreted planetesimals fall to the core. Also, it was found that a substantial amount of ices could stay in the envelope (Iaroslavitz \& Podolak 2007).

Both of these formation models, although advanced in many ways, do not follow throughout the evolution the fate of the high- $Z$ material. For example, accreted planetesimals could either fall to the center or evaporate in the envelope and mix with the gas, grains can slowly settle to the center, and high- $Z$ material from the core could be mixed back up by core erosion. These processes (and more) suggest that the final internal structure of a giant planet is not well constrained, and cannot be directly related to the formation mechanism.

In the context of extrasolar giant planets, one must therefore think in terms of global or bulk composition, instead of planetary structure or atmospheric enrichment. Even for Jupiter and Saturn, for which the gravitational moments are measured, the internal structures are not well determined, and are often dependent on model assumptions, equations of state, etc. At present, the available information on the compositions of extrasolar giant planets is insufficient for discriminating between the two models for giant planet formation. Providing predictions of the internal structures, and atmospheric properties of giant planets is still very challenging for formation models.

\section{References}

Bodenheimer, P., Grossman, A. S., Decampli, W. M., Marcy, G., \& Pollack, J. B. 1980, Icarus, 41, 293

Boss, A. P. 1997, Science, 276, 1836

Decampli, W. M. \& Cameron, A. G. W. 1979, Icarus, 38, 367

Durisen, R. H., Boss, A. P., Mayer, L.;Nelson, A. F., Quinn, T., \& Rice, W. K. M. 2007, in Protostars and Planets V, B. Reipurth, D. Jewitt, and K. Keil (eds.), Univ. of Arizona Press, Tucson, 607

Guillot, T. 2008, in Nobel Symposium 135, Physica Scripta, 130, 014023

Helled, R. \& Bodenheimer, P. 2010, Icarus, 207, 503

Helled, R. \& Bodenheimer, P. 2011, Icarus, 211, 939

Helled, R., Podolak, M., \& Kovetz, A. 2006, Icarus, 185, 64

Helled, R., Podolak, M., \& Kovetz, A. 2008, Icarus, 195, 863

Helled, R. \& Schubert, G. 2008, Icarus, 198, 156

Helled, R. \& Schubert, G. 2009, ApJ, 697, 1256

Iaroslavitz, E. \& Podolak, M. 2007, Icarus, 187, 600

Lissauer, J. J. 1987, Icarus, 69, 249

Lissauer, J. J. 1993, ARAEAA, 31, 129

Lissauer, J. J. \& Stevenson, D. J. 2007, in Protostars and Planets V, B. Reipurth, D. Jewitt, and K. Keil (eds.), University of Arizona Press, Tucson, 591

Lissauer, J. J., Hubickyj, O., D'Angelo, G., \& Bodenheimer, P. 2009, Icarus, 199, 338

Marois, C., Macintosh, B., Barman, T., et al. 2008, Science, 322, 1348

Movshovitz, N., Bodenheimer, P., Podolak, M., \& Lissauer, J. J. 2010, Icarus, 209, 616

Pollack, J. B., Hubickyj, O., Bodenheimer, P., Lissauer, J. J., Podolak, M., \& Greenzweig, Y. 1996, Icarus, 124, 62

Safronov, V. S. 1969, Evolution of the Protoplanetary Cloud and the Formation of the Earth and Planets (Nauka, Moscow) 